07

\title{
Влияние малоамплитудных осцилляций нагрузки на наноконтактные характеристики материалов в процессе наноиндентирования
}

\author{
(С) Ю.И. Головин, В.В. Коренков, С.С. Разливалова \\ Научно-исследовательский институт „Нанотехнологии и наноматериалы“ \\ Тамбовского государственного университета им. Г.Р. Державина, \\ Тамбов, Россия \\ E-mail: yugolovin@yandex.ru
}

(Поступила в Редакцию 25 октября 2016 г.)

\begin{abstract}
Критически проанализирован метод непрерывного измерения контактной жесткости, позволяющий непрерывно определять физико-механические свойства исследуемых материалов в процессе внедрения индентора под действием медленно растущей нагрузки при наноиндентировании. Границы применимости этого подхода к характеризации определяются условиями тестирования, при которых дополнительное наложение малоамплитудных осцилляций не влияет на механизмы, кинетику и величину пластической деформации материала под индентором. На примере материалов с аморфной структурой, ГЦК- и ОЦК-решетками показано, что различные методы определения наноконтактных характеристик материалов обладают разной чувствительностью к малоамплитудным осцилляциям нагрузки. Определены значения амплитуд осцилляций и диапазон глубин отпечатка, для которых влиянием осцилляций можно пренебречь. Выявлены закономерности влияния осцилляций на поведение контактных (локальных) характеристик исследованных материалов в закритических режимах.
\end{abstract}

Работа выполнена при поддержке Российского научного фонда (грант № 15-19-00181).

DOI: 10.21883/FTT.2017.06.44483.398

\section{1. Введение}

В настоящее время метод Оливера-Фарра (ОРМ), разработанный для извлечения механических характеристик материала из первичных данных наноиндентирования (NI) в виде исходных $P$ - $h$-диаграмм, регистрируемых в процессе глубиночувствительного индентирования, является широко распространенным и апробированным на широком круге материалов [1-5]. Здесь $P$ - сила вдавливания индентора, $h$ - глубина его погружения в материал. Эта наиболее продвинутая методика обработки „сырых“ данных принята стандартом РФ ГОСТ Р8.748-2011 и международным стандартом ISO-14577 [6] в качестве количественного метода характеризации физико-механических свойств твердых тел в нано- и микрошкалах. Однако еще более значительно расширить объем информации о деформационном отклике материала в приповерхностном слое позволяет метод непрерывного измерения контактной жесткости (continuous stiffness measurement, CSM) [7-10]. Запатентованный еще в 1989 г. [11], он заключается в наложении на исходную нормальную нагрузку малоамплитудного гармонического возмущения $P(t)=P_{a} \sin \omega t$, где $P_{a}-$ амплитуда добавочной гармонической силы, $\omega-$ ее частота. Благодаря наложению малоамплитудных осцилляций метод CSM (в отличие от ОРМ) позволяет непрерывно регистрировать механические свойства исследуемых материалов по мере погружения индентора любой геометрии, а также определять упругие и демпфирующие характеристики зоны деформации образца в процессе нагружения, а не после разгрузки индентора [12-15].
Опция CSM дает возможность изучать механические характеристики в пленках толщиной $10 \mathrm{~nm}$ и менее [16], отдельных фазах композитных материалов, многослойных покрытиях [17], а также изменение механических свойств с ростом размеров деформированной области (т.е. размерные эффекты); проводить десятки и сотни тестов на сопротивление пластической деформации и усталости в наношкале на одном образце [18]; тестировать на износ отдельные детали микро-/наноэлектромеханических систем [19] и т.д.

По духу и принципам метод CSM является локальным аналогом методов динамического механического анализа [20] и внутреннего трения [21], которые более полувека используются как тончайший инструмент исследования динамики атомных дефектов и релаксационных процессов в различных материалах на макрообразцах. Как известно, во внутреннем трении есть как амплитудно-независимая область, так и амплитудно-зависимая. Это означает, что до некоторой критической амплитуды осцилляций нагрузки/деформации атомарная дефектная структура не претерпевает необратимых изменений в процессе испытаний. Такие испытания можно считать „неразрушающими“. Однако при превышении некоторой критической величины амплитуды осцилляций начинается необратимая перестройка структуры (например, размножение дислокаций, их перемещение на большие расстояния, изменение структуры субграниц и границ зерен и т.п.). В условиях одновременного действия статической и/или медленно нарастающей нагрузки и дополнительной осциллирующей эти явления вызывают акустопластиче- 
ский эффект, выражающийся в уменьшении предела текучести, коэффициента деформационного упрочнения и др. [22-24]. Аналогичные явления можно ожидать и при одновременном действии локальной медленно нарастающей нагрузки при NI и добавленных к ней малоамплитудных осцилляций. Поскольку метод CSM рассматривается как чисто измерительный, закономерно возникает вопрос: до какой амплитуды зондирующей осцилляции его можно считать не влияющим на результаты измерений, т. е. „неразрушающим“? Ясно, что это зависит от физико-механических свойств материала, скорости нарастания основной нагрузки и других условий эксперимента.

Существенна и другая сторона вопроса. Невзирая на все технические ухищрения, направленные на подавление малоамплитудных вибраций, последние являются неотъемлемым атрибутом любых механических испытаний. Механическая обработка, шлифовка и полировка, весь спектр трибологических процессов также не обходятся без вибрационного вклада в нагружающее усилие [25]. При этом поведение дефектной структуры материалов в локальных сильнодеформированных объемах под инструментом/индентором в присутствии низкочастотных виброакустических колебаний изучено недостаточно. Правда, в последнее время появились экспериментальные свидетельства того, что физикомеханические свойства ряда металлических материалов $[4,7,12,23,24,26-28]$ при локальном нагружении могут значительно измениться при наложении дополнительных гармонических осцилляций нагрузки.

Например, в [29] было рекомендовано уменьшить амплитуду осцилляции до минимальной, равной $0.5 \mathrm{~nm}$, для исследования механических свойств пластичных металлов с помощью CSM либо провести коррекцию данных по нагрузке, смещению и жесткости. Следует отметить, что применение низких амплитуд осцилляции не всегда подходит для исследования поведения механических характеристик материалов в наношкале. Так, применение осцилляций с амплитудами $z=0.5-1 \mathrm{~nm}$ вызывало сильное снижение отношения сигнал/шум, что вело к большим погрешностям при извлечении характеристик напряжение-деформация в процессе индентирования сферой (радиусами 1 и $100 \mu \mathrm{m}$ ) [30]. В качестве возможных причин наблюдаемого разупрочнения в пятне контакта наряду с влиянием гармонической нагрузки на дефектную структуру материала называют, в частности, и методологические погрешности [7,29]. При этом методология CSM продолжает развиваться и совершенствоваться по пути регистрации второй гармоники [31] и за счет этого дальнейшей минимизации погрешности [32]. В большинстве случаев объектом исследования являлись мягкие и пластичные металлы с гранецентрированной кубической (ГЦК) решеткой, в то время как для жестких материалов влияние малоамплитудной осцилляции нагрузки на локальные механические свойства изучено мало.
Целью настоящей работы является определение диапазона нагрузок/деформаций, при которых метод CSM можно считать „неразрушающим“ в пределах точности измерений, т.е. не влияющим на механизмы, кинетику и величину пластической деформации под индентором, для материалов, обладающих различной структурой и значениями нанотвердости: аморфного плавленого кварца, монокристаллов арсенида галлия, монокристаллов высокочистых молибдена $(99.99 \%)$ и вольфрама $(99.99 \%)$, монокристаллов высокочистого алюминия $(99.99 \%)$. В запороговых режимах исследование направлено на определение характера влияния дополнительных осцилляций индентора на локальные механические свойства и выявление их зависимости от соотношения амплитуды осцилляций и накопленной деформации.

\section{2. Аппаратура, материалы и подготовка образцов}

Эксперименты проводились на приборе NanoIndenter G200 фирмы Agilent с помощью алмазного индентора Берковича с радиусом притупления вершины $R=20 \mathrm{~nm}$ в диапазоне нагрузок $0.01-100 \mathrm{mN}$. Тестирование осуществлялось двумя основными модами нагружения: a) традиционной квазистатической без добавления осцилляции, b) посредством метода CSM с наложением дополнительной осцилляции смещения индентора амплитудой $z=0.5-8 \mathrm{~nm}$ и частотой $45 \mathrm{~Hz}$. Во время проведения всех экспериментов скорость относительной квазистатической деформации поддерживалась постоянной $\left(\varepsilon=0.05 \mathrm{~s}^{-1}\right)$ с помощью цепей обратной связи в приборе, что позволяло исключить влияние скоростного фактора на величину твердости [33]. Максимальная глубина отпечатков в контрольных образцах (для которых $z=0)$, устанавливаемая протоколом испытания, составляла $25,50,100,200,500,1000,1500$ и $2000 \mathrm{~nm}$. Все отпечатки наносились на расстоянии не менее $50 \mu \mathrm{m}$ друг от друга во избежание их взаимного влияния. Значения твердости $H$ и модуля Юнга $E$ усреднялись по данным десяти отпечатков. Сканирование остаточных отпечатков производилось на сканирующем зондовом микроскопе diInnova (Veeco, USA) в полуконтактном режиме атомно-силовой микроскопии (AFM).

В качестве объекта исследования выбрано несколько репрезентативных представителей различных классов материалов: a) мягкий и пластичный металл с ГЦК-решеткой (Al); b) жесткий полупроводник $\mathrm{GaAs}$; c) аморфный плавленый кварц, обычно используемый для калибровки наноиндентометров; d) твердые металлы с объемно центрированной кубической (ОЦК) решеткой, к которым относятся W и Мо.

Монокристаллический Al (99.99\%) взят как материал с хорошо изученной дефектной структурой, многократно исследованный методами традиционного NI [26-28]. Поверхность образцов Al размером $10 \times 10 \times 3 \mathrm{~mm}$ подготавливалась механической шлифовкой с помощью аб- 
разива $\mathrm{SiC}$ (1200 grit) и полировкой алмазной пастой на устройстве для пробоподготовки „Buehler Vector Power Grid“. После механической полировки поверхностный слой подвергался электрохимической полировке в течение $3 \mathrm{~min}$ при плотности тока $1 \mathrm{~A} / \mathrm{cm}^{2}$ в $10 \%$ раствоpe $\mathrm{HCl}$ в этаноле для устранения приповерхностного деформационно-упрочненного слоя.

Нелегированные образцы монокристаллического GaAs (99.999\%, Sigma Aldrich) с ориентацией (001) были нарезаны в виде параллелепипедов размером $10 \times 5 \times 1 \mathrm{~mm}$ и последовательно полировались порошком $\mathrm{Al}_{2} \mathrm{O}_{3}$ с размером частиц 5,1 и $0.3 \mu \mathrm{m}$.

Плавленый кварц намеренно выбран в качестве тестируемого материала, поскольку при комнатной температуре он является линейно упругим материалом с фазовым углом между приложенной силой и деформацией, очень близким к нулю [34].

Образцы молибдена $(50 \times 50 \times 3 \mathrm{~mm}$, чистоты $99.95 \%)$ и вольфрама $(6 \times 12 \times 3 \mathrm{~mm}$, чистоты $99.97 \%)$ были изготовлены механической прокаткой (Мо) или путем плавления большого слитка в атмосфере инертного газа $(\mathrm{W})$. Образцы также полировались по стандартной методике с помощью „Buehler Vector Power Grid“ до зеркального блеска. Конечная шероховатость поверхности образцов измерялась методом AFM („tapping mode“ на площадке $4 \mu \mathrm{m}^{2}$. Средняя квадратическая величина шероховатости для всех образцов не превосходила $2 \mathrm{~nm}$.

\section{3. Методика извлечения экспериментальных данных}

Наноконтактные характеристики твердости $H$ и модуля Юнга $E$ рассчитывались следующими способами.

1. Методом Оливера-Фарра [1-4] путем анализа разгрузочной ветви $P$-h-диаграммы. В этом случае интегральная жесткость $S=d P / d h$ контакта индентор-

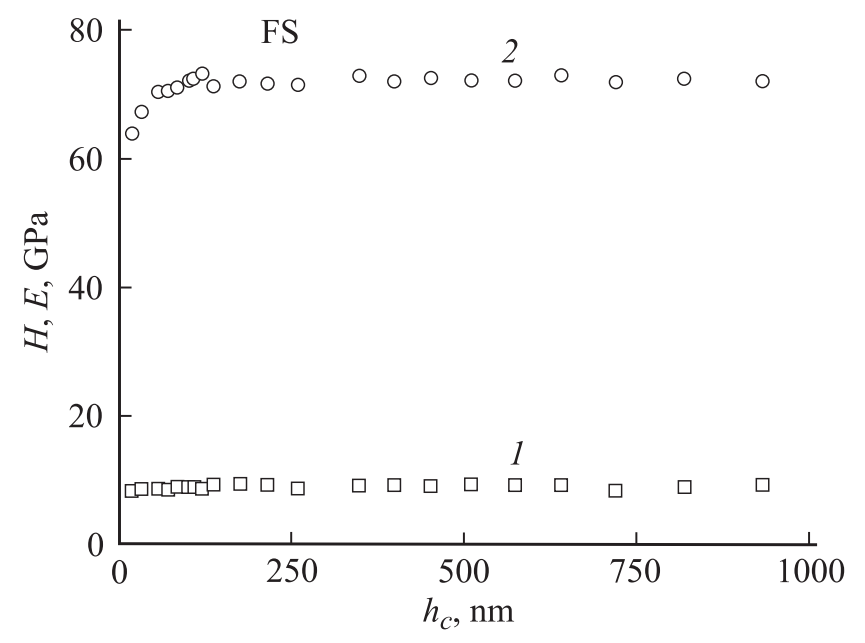

Рис. 1. Зависимости твердости $H(1)$ и модуля упругости $E(2)$ от контактной глубины $h_{c}$ для плавленого кварца $(\mathrm{FS})$. образец определяется наклоном кривой разгружения, которая аппроксимируется степенной функции вида

$$
P=k_{i}\left(h_{\max }-h_{r}\right)^{m},
$$

откуда следует

$$
S=d P / d h=m B\left(h_{\max }-h_{r}\right)^{m-1},
$$

где $k_{i}, m, B$ - материальные константы, $h_{r}$ - глубина остаточного отпечатка. Знание величины $S$ позволяет вычислить истинную глубину отпечатка индентора

$$
h_{c}=h_{\max }-\varepsilon P_{\max } / S,
$$

где $\varepsilon-$ коэффициент, зависящий от формы индентора $(\varepsilon=0.72$ для конического индентора или $\varepsilon=0.75$ для параболоида вращения) [4].

Макроскопическая твердость $H$ определялась традиционным выражением

$$
H=P_{\max } / A_{c} .
$$

Величина проекции площади контакта $A_{c}$ определялась исходя из соображений геометрического подобия индентора и его отпечатка в материале

$$
A_{c}=24.56 h_{c}^{2} \text {. }
$$

При малых нагрузках (и соответственно малых $h_{c}$ ) учитывалось, что форма кончика индентора неидеальна, и формула (5) заменялась функцией площади индентора, которую можно определить различными способами [1,2,35-37]. В настоящей работе функция площади индентора Берковича определялась по стандарту ISOZ-14577 посредством нанесения серии отпечатков в диапазоне нагрузок $0.06-500 \mathrm{mN}$ на плавленый кварц, модуль Юнга которого полагался неизменным и равным $E_{\mathrm{FS}}=72 \mathrm{GPa}$. Для использовавшегося в работе индентора найденная экспериментально функция площади имела вид

$$
A_{c}=24.43 h_{c}^{2}+49.92 h_{c}+0.387 h_{c}^{0.5} .
$$

При таком выборе $h_{c}$ предполагалось, что (6) действует до такой глубины отпечатка, начиная с которой искажением формы кончика индентора можно пренебречь. Зависимости $H$ и $E$ плавленого кварца от глубины, полученные при калибровке данного индентора, приведены на рис. 1.

2. Методом CSM действительная часть гармонической жесткости контакта определяется для каждого периода осцилляции следующим выражением:

$$
z / P_{0}=1 / S=1 /\left(\left(K_{i}-m \omega^{2}\right)^{2}+\left(D_{i} \omega\right)^{2}\right)^{0.5},
$$

где $K_{i}$ - жесткость подвеса индентора, $D_{i}-$ коэффициент затухания, $m$ - масса подвижных частей измерительной головки. Основные упругие и демпфирующие константы самого прибора Agilent G200 NanoIndenter ${ }^{\circledR}$ известны: $K_{i}=92.02 \mathrm{~N} / \mathrm{m}, \quad m=11.6 \mathrm{~g}$, 
$D_{i}=2.66 \mathrm{~N} \cdot \mathrm{s} / \mathrm{m}[7-10]$, так что последовательное применение выражений (2), (3) и (6) позволяет находить твердость в любой точке диаграммы нагружения. Как следствие, при этом происходит сканирование твердости по глубине отпечатка в единственном испытании, что заменяет целую серию испытаний методом традиционного NI [7,38,39].

3. Твердость определялась также с помощью OРM $[1,2]$ при анализе разгрузочной ветви диаграммы $P-h$ вблизи $P_{\max }$ (аналогично методу 1 только с добавлением малоамплитудной осциллирующей компоненты на стадии нагружения). Этот метод фактически позволяет с помощью одной диаграммы нагружения сравнить значения твердости, рассчитанные методами 1 и 2.

4. Площадь проекции отпечатков измерялась прямым методом обработки изображений отпечатков, полученных с помощью AFM в полуконтактном режиме [36]. По этим данным была рассчитана твердость для $z=0$, которая сравнивалась с рассчитанной на основе ОРМ.

\section{4. Результаты}

Типичные зависимости нагрузка-смещение индентора при его погружении в плавленый кварц с наложением малоамплитудных осцилляций смещения с $z=0.5-6 \mathrm{~nm}$ и без него представлены на рис. 2. Из этого рисунка следует, что величина нагрузки, необходимая для внедрения индентора на фиксированную глубину, последовательно уменьшалась с ростом амплитуды осцилляции смещения: на глубине $60 \mathrm{~nm}$ она составляла $P \approx 0.55 \mathrm{mN}$ при $z=0, P=0.53 \mathrm{mN}$ при $z=0.5 \mathrm{~nm}$, $P=0.48 \mathrm{mN}$ при $z=2 \mathrm{~nm}, P=0.42 \mathrm{mN}$ при $z=6 \mathrm{~nm}$. При фиксированной нагрузке это приводит в свою очередь к росту глубины погружения индентора $h$ с увеличением $z$.

В качестве меры влияния наложенных осцилляций на локальную деформацию под индентором принята

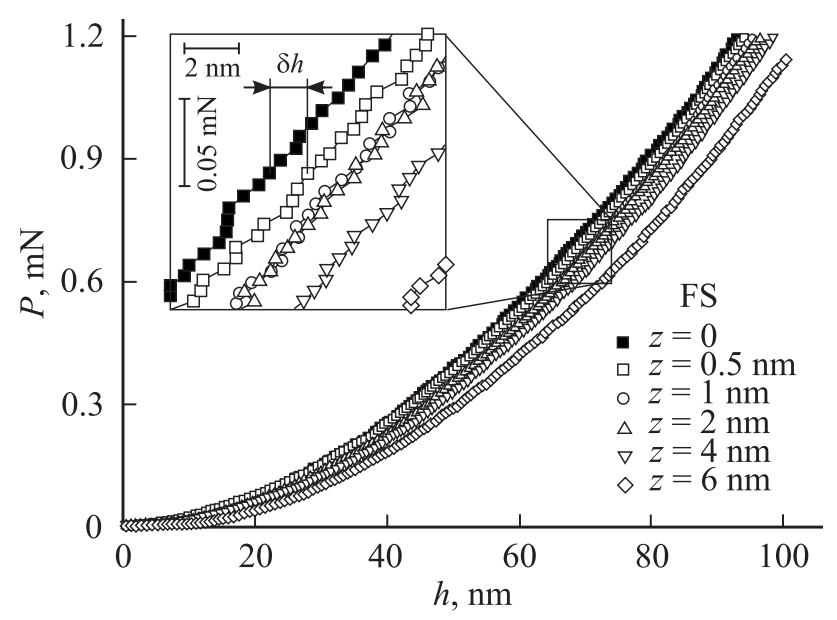

Рис. 2. Типичные ветви погружения индентора в плавленый кварц при наложении дополнительной осцилляции смещения с амплитудами от 0.5 до $6 \mathrm{~nm}$ и без него. разность глубин $\delta h$ между кривыми нагружения с наложением дополнительной осцилляции смещения $(z>0)$ и без него $(z=0)$ в каждой точке с одинаковой нагрузкой (вставка на рис. 2). Здесь $\delta h$ является количественной характеристикой дополнительной деформации под действием осцилляций. Вдавливание индентора Берковича в плавленый кварц, выбранный как калибровочный материал, выполнялось силой, достигающей в конце нагружения величины $P_{\max }$ от 0.15 до $440 \mathrm{mN}$. Зависимости твердости и модуля упругости материала показаны на рис. 1. Модуль упругости плавленого кварца оставался примерно постоянным во всем диапазоне глубин тестирования (равным $\sim 72 \mathrm{GPa}$ ). Величина твердости приблизительно соответствовала справочным значениям, равным $H=9 \mathrm{GPa}$. Данный факт может свидетельствовать о том, что калибровка индентора была проведена корректно.

Зависимости разности $\delta h$ от глубины внедрения индентора и амплитуды осцилляции смещения показаны на рис. 3 для монокристаллов плавленого кварца $(a)$, алюминия $(b)$, вольфрама $(c)$, молибдена $(d)$ и арсенида галлия $(e, f)$. Из рис. 3 со всей очевидностью следует, что в разных материалах величина $\delta h$ ведет себя по-разному. Но вместе с тем есть и некоторые элементы сходства.

Прежде всего можно выделить несколько областей характерного поведения $\delta h$ в зависимости от глубины $h$. Сначала следует отметить, что $\delta h$ при значениях амплитуды осцилляции $z=0.5$ и $1 \mathrm{~nm}$ для всех материалов принимает примерно постоянные значения во всем диапазоне глубин исследования. В первой области происходит резкий рост $\delta h$ на первых $5 \mathrm{~nm}$ внедрения индентора. Данная область, как правило, имела место при больших амплитудах осцилляции смещения для всех материалов: $\mathrm{FS}$ (при $z=4$ и $6 \mathrm{~nm}$ ), Al (при $z=2$ и $4 \mathrm{~nm}$ ), Мо (при $z=4$ и $8 \mathrm{~nm}$ ), W (при $z=2,4$ и $8 \mathrm{~nm}$ ). Возможная причина резкого роста $\delta h-$ неточность в определении прибором момента контакта индентора с поверхностью образца. Для второй области характерен рост $\delta h$ на стадии упругопластической деформации (сразу после скачка деформации на Al) до достижения максимальных значений (данная область наблюдалась для всех металлов при $z \geq 2 \mathrm{~nm})$. Наконец, в третьей области происходит постепенное снижение $\delta h$ с ростом глубины индентирования $h$ после достижения максимальных значений во второй области (данная область имела место при больших амплитудах осцилляции $z>4 \mathrm{~nm}$ для всех материалов).

Рассмотрим более подробно поведение $\delta h$ с ростом глубины для плавленого кварца и алюминия. Резкий рост $\delta h$ в случае плавленого кварца происходил при больших амплитудах осцилляции $(z=4-6 \mathrm{~nm})$ на ранней стадии внедрения индентора в материал (непосредственно на первых $5 \mathrm{~nm}$ ) (первая область). Вплоть до $h \approx 30 \mathrm{~nm}$ зависимости $\delta h$ были параллельными и не демонстрировали роста, затем при $h=30 \mathrm{~nm}$ начинался рост $\delta h$, увеличивающийся с ростом амплитуды осцилляции смещения (вторая область). Таким образом, можно 

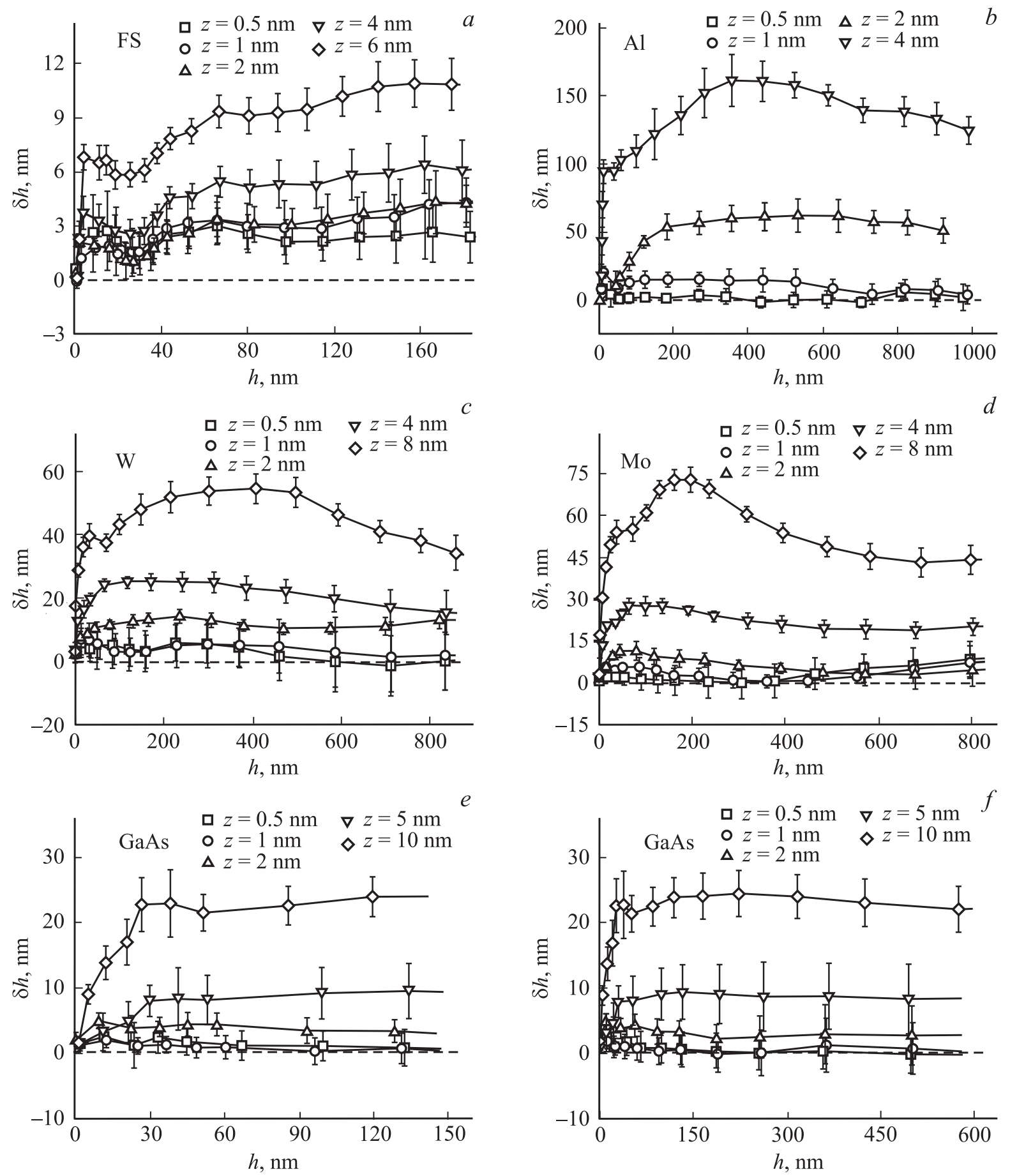

Рис. 3. Зависимости добавочного смещения $\delta h$ от глубины $h$, рассчитанные из ветвей нагружения индентора с наложением дополнительной осцилляции смещения при погружении в плавленый кварц $(a)$, монокристаллы алюминия $(b)$, вольфрама $(c)$, молибдена $(d)$, арсенида галлия $(e, f)$.

предположить, что на глубине $h \sim 30 \mathrm{~nm}$ происходил переход от упругой к упругопластической деформации плавленого кварца. В области чисто упругой деформации $(h<30 \mathrm{~nm})$ дополнительная осцилляция не оказывала влияния на поведение сильнодеформированного объема материала под индентором, в области упругопластической деформации $(30<h<180 \mathrm{~nm})$ влияние осцилляции было в разы слабее, чем в кристаллах Al, GaAs, Мо и W.

Для $\mathrm{Al}$ величина $\delta h$ принимала относительно постоянные значения при $z=0.5$ и $1 \mathrm{~nm}$ во всем диапазоне глубин тестирования. За ростом $\delta h$ в первой области (на первых $5 \mathrm{~nm}$ внедрения индентора) следовала область чисто упругой деформации, когда осцилляция 

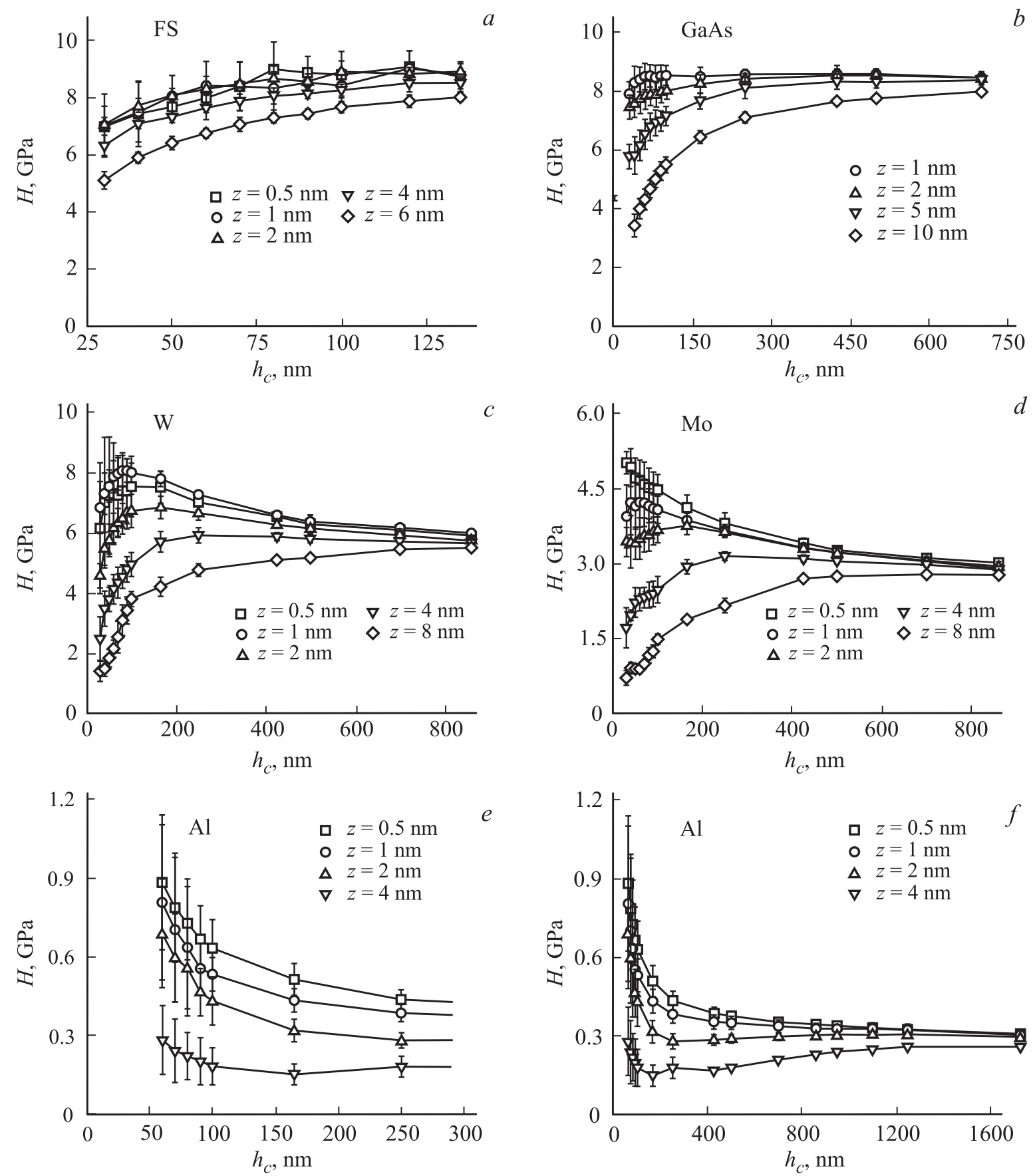

Рис. 4. Зависимости твердости $H$ от контактной глубины $h_{c}$ при погружении индентора с наложением дополнительной осцилляции смещения в плавленый кварц $(a)$, монокристалл арсенида галлия $(b)$, вольфрама $(c)$, молибдена $(d)$ и алюминия $(e, f)$.

не оказывала влияния на поведение материала. При $h \geq 50 \mathrm{~nm}$ сразу после скачкообразной деформации в области пластической деформации для $z=2$ и $4 \mathrm{~nm}$ происходил существенный рост $\delta h$ (вторая область), далее для $z=2 \mathrm{~nm}$ величина $\delta h$ была постоянной, а для $z=4 \mathrm{~nm}$ постепенно снижалась (третья область) при $h>450 \mathrm{~nm}$.

Для монокристаллов GaAs и Al переход от упругой к упругопластической деформации происходил скачкообразно и сопровождался быстрым погружением инден- тора в материал под действием постоянной нагрузки (на глубине внедрения индентора примерно 25-30 nm для GaAs и 8-15 nm для монокристаллов Al при квазистатическом нагружении). Природа скачков на ранней стадии внедрения индентора в GaAs и Al была хорошо изучена и связывалась с гомогенным зарождением дислокаций в материале под индентором [40-45].

На зависимостях $\delta h$ от глубины для GaAs можно выделить уже четыре характерные области. Для первой области, как и в плавленом кварце, происходил резкий 
рост $\delta h$ на первых $5 \mathrm{~nm}$ внедрения индентора для больших амплитуд осцилляций $(z=5,10 \mathrm{~nm})$. Во второй области, соответствующей чисто упругой деформации, был заметен слабый линейный рост $\delta h$ с ростом глубины индентирования $h$. Рост $\delta h$ происходил до критической глубины, при которой наблюдался скачок перехода от упругой к упругопластической деформации. Сразу после этого скачка $\delta h$ демонстрировала незначительный рост только при $z=10 \mathrm{~nm}$ (третья область), а затем постепенно снижалась до $h=600 \mathrm{~nm}$ (четрвертая область). Для меньших $z$ величина $\delta h$ не демонстрировала существенного роста после скачка деформации и принимала примерно постоянные значения до глубин $h=700 \mathrm{~nm}$.

Поведение $\delta h$ для ОЦК-металлов (рис. $3, c, d$ ) не имело существенных качественных особенностей по сравнению с ГЦК-металлами. Здесь также можно выделить две области: $0.5-1 \mathrm{~nm}$ для $\mathrm{W}$ и $0.5-2 \mathrm{~nm}$ для Мо, в пределах которых влиянием дополнительных осцилляций смещения можно пренебречь ввиду их малости. В то же время дополнительное смещение $\delta h$ для $\mathrm{W}$ и Мо по абсолютной величине остается в 2.5-3 раза меньше, чем в ГЦК-металлах на той же глубине.

Еще одной величиной, чувствительной к действию малоамплитудных гармонических осцилляций, является твердость $H$. Как следует из рис. 4 , с ростом $z$ снижение величины $H$, определяемой в каждой точке кривой нагружения методом CSM, возрастало для GaAs, W, Mo и Al и было максимальным при наибольшей амплитуде осцилляции на контактных глубинах $h_{c}<300 \mathrm{~nm}$. Как следует из рис. $4, a$, повышение амплитуды дополнительной осцилляции от $z=0.5$ до $4 \mathrm{~nm}$ не вызывало значительного снижения $H$ плавленого кварца в диапазоне контактных глубин от 30 до $135 \mathrm{~nm}$. В то же время при $z=6 \mathrm{~nm}$ происходило снижение $H$ приблизительно на $30 \%$ при $h_{c}=30 \mathrm{~nm}$ по сравнению с $H$ при $z=0.5 \mathrm{~nm}$. С дальнейшим увеличением $h_{c}$ до $135 \mathrm{~nm}$ разница в твердости уменьшалась и становилась незначительной. Следует отметить, что $\delta h$ при $z=6 \mathrm{~nm}$ была намного больше, чем при $0.5,1,2$ и $4 \mathrm{~nm}$ (рис. 3,a), и это приводило к снижению $H$.

Во всем диапазоне $h_{c}$ (от 30 до $700 \mathrm{~nm}$ ) твердость GaAs при $z=1 \mathrm{~nm}$ принимала практически постоянные значения, равные $8.5 \pm 0.5 \mathrm{GPa}$, вариативность значений $H$ на начальных этапах нагружения $(30-100 \mathrm{~nm})$ не превышала $1 \mathrm{GPa}$. Снижение твердости было значительным на контактных глубинах $h_{c}<300 \mathrm{~nm}$ при $z=5$ и $10 \mathrm{~nm}$, что в принципе согласовалось со значительным ростом $\delta h$ для данных амплитуд осцилляции (рис. $3, e, f)$.

Твердость в наношкале сильно снижалась для $\mathrm{W}$ и $\mathrm{Al}$ уже при $z=2 \mathrm{~nm}$. Для $\mathrm{Al}$ она была близка к характерным значениям микротвердости $(0.3 \mathrm{GPa})$ при $h_{c}>150 \mathrm{~nm}$. Следует отметить, что осцилляция с большими амплитудами смещения может применяться для определения твердости на больших контактных глубинах: более 750-800 nm для GaAs, Мо и W и более $1500 \mathrm{~nm}$ для $\mathrm{Al}$.

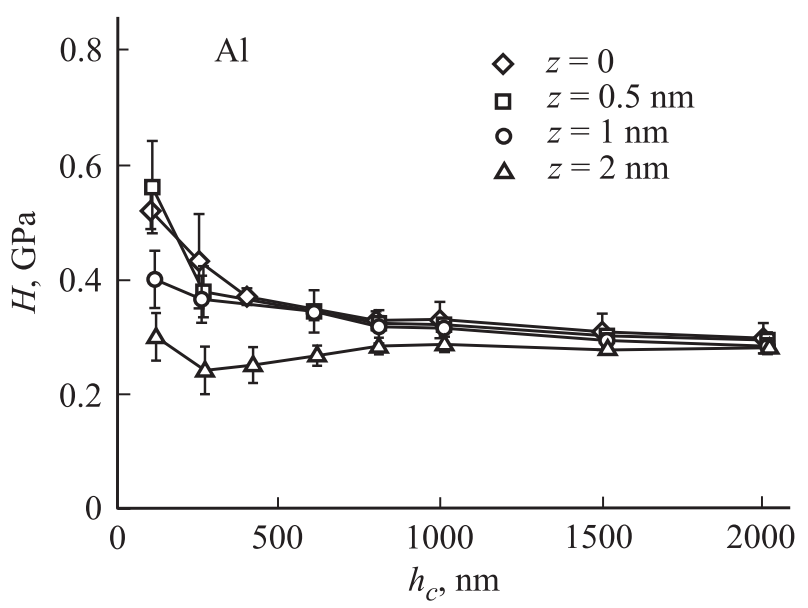

Рис. 5. Твердость алюминия, определенная с помощью ОРМ с наложением дополнительных осцилляций с амплитудами $z=0.5,1$ и $2 \mathrm{~nm}$, а также при нагружении без наложения дополнительной осцилляции смещения $(z=0)$.

Как видно из рис. 4, твердость как ГЦК-, так и ОЦК-металлов уменьшалась с ростом контактной глубины и демонстрировала размерный эффект. О размерном эффекте в алюминии известно давно, причем он имел место независимо от способа предварительной обработки: в монокристаллах [46,47], высокочистых (99.99 и 99.9999\%) полированных механически поликристаллах алюминия [48], а также в поликристаллах, не подвергавшихся предварительной механической обработке [49]. Применение предварительной механической полировки приводило к более высоким регистрируемым значениям $H$, нежели применение электрохимической, однако не исключало наличия размерного эффекта в твердости [50].

Размерный эффект в твердости исследовался и для моно- и поликристаллов ОЦК-металлов [51]. Твердость монокристалла вольфрама зависела не только от величины приложенной нагрузки, но и от ориентации монокристалла и ориентации индентора относительно осей кристалла [52]. При этом твердость монокристаллов $\langle 100\rangle$ вольфрама демонстрировала размерный эффект на очень малых глубинах внедрения при индентировании коническими инденторами [53], а также инденторами Берковича и Бирбаума (углом куба) [54].

Предложены две основные теории, интерпретирующие наблюдаемый размерный эффект в твердости на глубинах $h<100 \mathrm{~nm}$. Модифицирование теории градиентной деформации, основанной на дислокационной модели Тэйлора и включающей максимально допустимую плотность геометрически необходимых дислокаций [55], позволяло объяснить появление размерного эффекта в наношкале. Результаты моделирования методом конечных элементов на основе предложенной модели показали хорошее согласие с экспериментальными данными о микро- и нанотвердости $\mathrm{MgO}$ и Ir. Альтернативная теория градиентной деформации (модель Гербериха и 

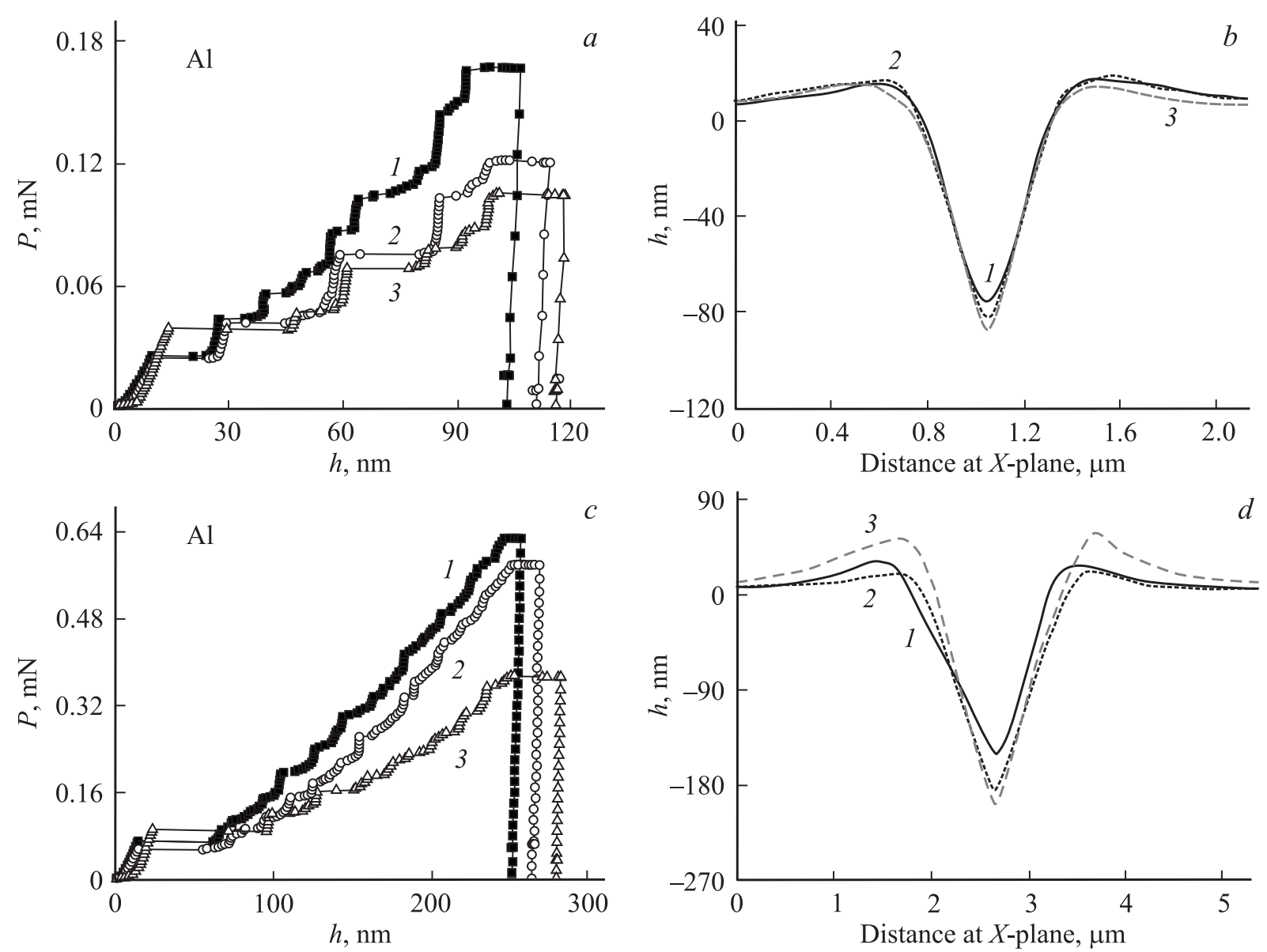

Рис. 6. Результаты испытания $\mathrm{Al}$ на нанотвердость. $a$ - типичные $P$ - $h$-диаграммы нагружения индентора при заданной глубине отпечатка $100 \mathrm{~nm}$, полученные при $z=0(1), 1(2), 2 \mathrm{~nm}(3) ; b-$ профили отпечатков при заданной глубине $100 \mathrm{~nm} ; c-$ типичные $P-h$-диаграммы нагружения индентора при заданной глубине отпечатка $250 \mathrm{~nm}$, полученные при $z=0(1), 1(2) 2 \mathrm{~nm}(3) ; d-$ профили отпечатков при заданной глубине $250 \mathrm{~nm}$.

др. [56]) базируется на анализе отношения работы по пластической деформации поверхности к работе по вытеснению объема материала. Обе модели не включают в рассмотрение влияние дополнительных осцилляций на проявления размерного эффекта в разных материалах.

Твердость Al при наложении осцилляции смещения с $z$ от 0.5 до $2 \mathrm{~nm}$ и без него была рассчитана с помощью ОРМ по разгрузочной ветви $P-h$-диаграммы (рис. 5). Для амплитуд осцилляции $z=0.5$ и $1 \mathrm{~nm}$ твердость при $100 \leq h_{c} \leq 2000 \mathrm{~nm}$ имела значение, близкое к твердости при $z=0$. Значительное расхождение в $H$ наблюдалось при амплитуде осцилляций $z=2 \mathrm{~nm}$ на глубинах менее $600 \mathrm{~nm}$. Так, для $h_{c}=250 \mathrm{~nm}$ снижение твердости составляло $\sim 45 \%$. При наложении осцилляции с $z=2 \mathrm{~nm}$ значения твердости в наношкале, определенные с помощью ОРМ, были близки к значениям микротвердости алюминия, как и в случае определения твердости в каждой точке кривой нагружения при $z=2 \mathrm{~nm}$ (рис. $4, e, f)$. Из этого следует, что наложение осцилляции с $z=2 \mathrm{~nm}$ приводит к значительному снижению твердости $\mathrm{Al}$ в наношкале, определенной как с помощью ОРМ, так и в каждой точке кривой нагружения методом CSM. Модуль упругости алюминия, рассчитанный из контактной жесткости (2) с помощью ОРМ, соответствовал справочным значениям $\sim 70 \mathrm{GPa}$

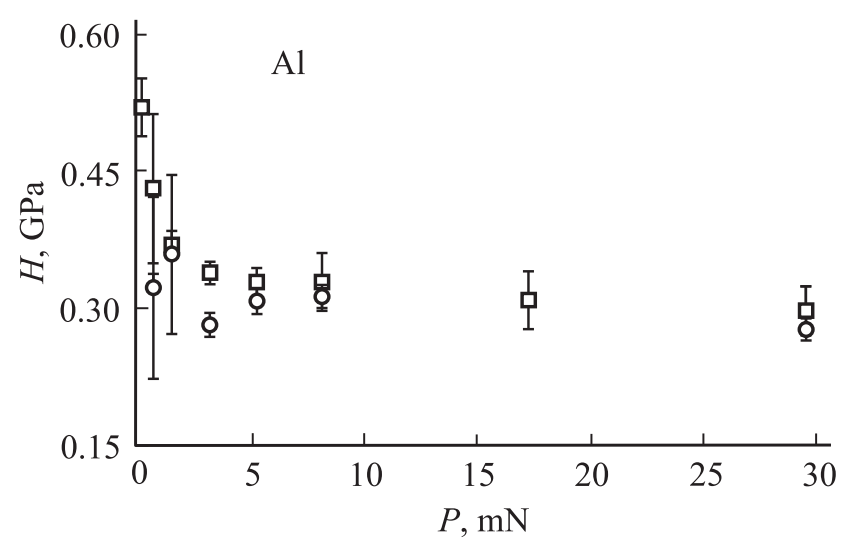

Рис. 7. Твердость, определенная путем прямого измерения площади отпечатков по изображениям, полученным методом AFM (кружки) и методом Оливера-Фарра (квадраты) с помощью $P$ - $h$-диаграмм при нагружении без наложения осцилляции. 

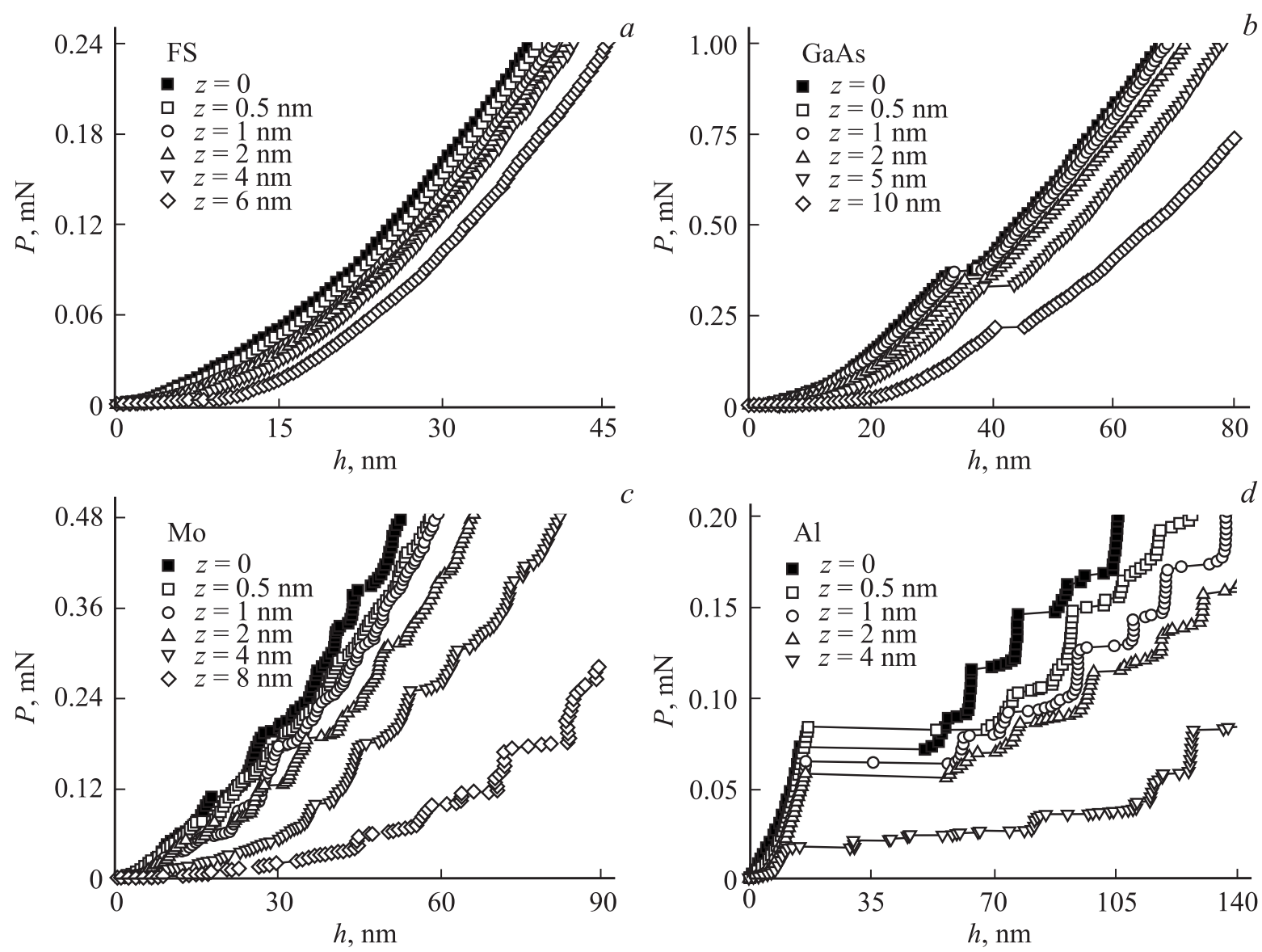

Рис. 8. Типичные $P$-h-диаграммы погружения индентора в плавленый кварц $(a)$, арсенид галлия $(b)$, молибден $(c)$ и алюминий $(d)$, полученные с наложением дополнительной осцилляции смещения на ветви нагружения и без него $(z=0)$.

во всем диапазоне $h_{c}$. Следует отметить, что модуль Юнга оказался практически нечувствительным к наложению дополнительной осцилляции с $z$ от 0.5 до $2 \mathrm{~nm}$. В совокупности с результатами по твердости это дает

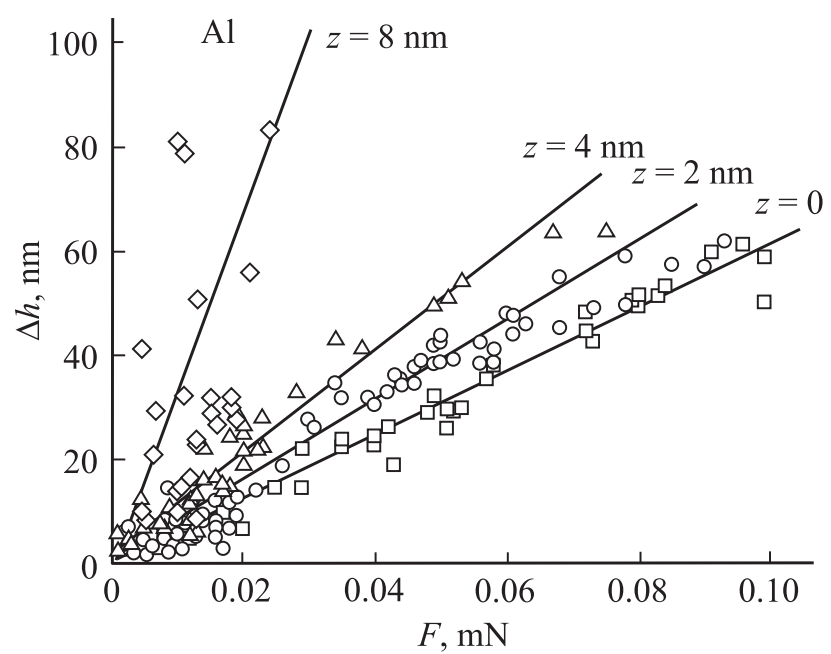

Рис. 9. Зависимость амплитуды первого скачка деформации $\Delta h$ от величины критической нагрузки $F$ при разных амплитудах осциллирующей компоненты нагрузки. возможность заключить, что осцилляция смещения с амплитудой до $0.5 \mathrm{~nm}$ может быть рекомендована для наноиндентирования методом CSM монокристаллов высокочистого алюминия и других ГЦК-металлов как не оказывающая существенного влияния на микромеханизмы пластичности под индентором в процессе индентирования в нано- и микрошкалах. Аналогичный результат ранее был получен для плавленого кварца (рис. 1), что дает основание для общности такого заключения. Для ОЦК-металлов нижняя граница независимости $H$ и $E$ от амплитуды осцилляций несколько выше и соответствует $z=1 \mathrm{~nm}$.

На рис. 6 изображены профили остаточных отпечатков в Al и соответствующие им диаграммы нагрузка-смещение при использовании метода CSM с наложением осцилляции смещения в диапазоне $z$ от 0 до $2 \mathrm{~nm}$. При еще большем увеличении $z(z>2 \mathrm{~nm})$ требовалась все меньшая нормальная нагрузка (рис. 6, $a$ ) для погружения индентора на глубину, равную $100 \mathrm{~nm}$ (задаваемую перед началом эксперимента условиями нагружения). Остаточные отпечатки индентора, при нагружении которого добавлялась осцилляция $z=2 \mathrm{~nm}$, были глубже в среднем приблизительно на $10 \mathrm{~nm}$. В случае первоначально заданной глубины погружения индентора 

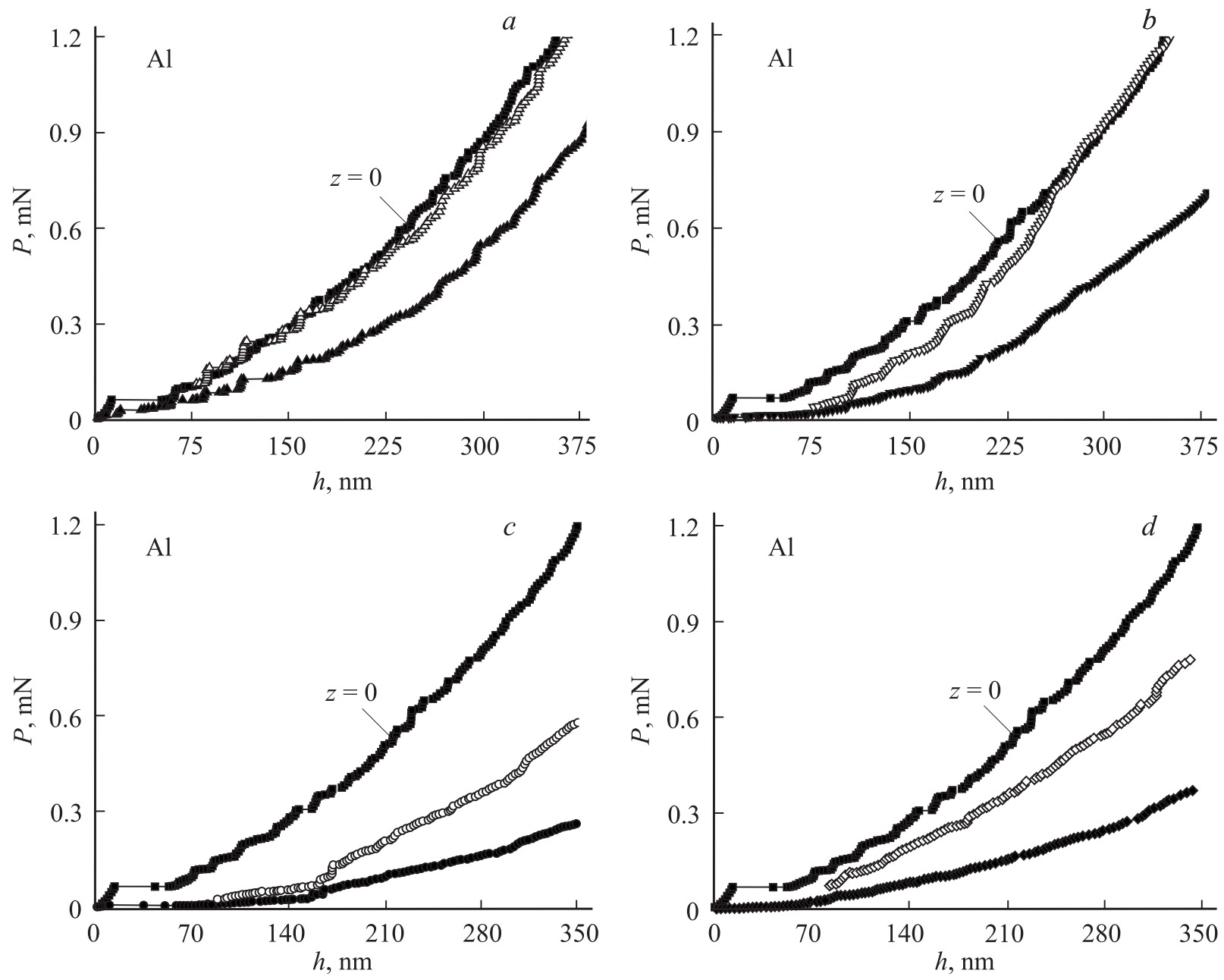

Рис. 10. Применение коррекции Оливера-Стрейдера-Фарра для ветвей нагружения индентора с наложением осцилляции с амплитудами $z=2(a), 4(b), 6(c), 8 \mathrm{~nm}(d)$. Темными символами обозначены типичные исходные кривые без применения коррекции, светлыми - отрезок ветви кривой нагружения, начинающийся после скачкообразной деформации на начальной стадии внедрения индентора, после применения коррекции. Приведена также типичная кривая нагружения при $z=0$.

$h=250 \mathrm{~nm}$ среднее различие в глубине составляло уже $\delta h \approx 60 \mathrm{~nm}$ при тех же условиях нагружения. Одновременно наблюдалось значительное увеличение высоты навалов вокруг отпечатков при $z=2 \mathrm{~nm}$, по сравнению с таковой в условиях нагружения при $z=0$ и $z=1 \mathrm{~nm}$.

Рост глубины отпечатков, полученных при нагружении с наложением осцилляции $z=1$ и $2 \mathrm{~nm}$, может служить подтверждением разупрочнения алюминия в наношкале. Площадь остаточных отпечатков, рассчитанная независимым методом для $z=0$ и $1 \mathrm{~nm}$, т.е. прямым измерением площади отпечатков на основе изображений, даваемых AFM [36], не показывает существенного различия в микро- и нанодиапазонах глубин (от 100 до $2000 \mathrm{~nm}$ ).

На рис. 7 показаны зависимости твердости от нагрузки, определенные двумя способами (ОРМ и прямым измерением площади в полуконтактном режиме AFM) при нагружении без использования дополнительной осцилляции смещения $(z=0)$. Полученные значения $H$ практически не различались при варьировании $h_{c}$ от 250 до $2000 \mathrm{~nm}$. Это согласуется с ранее полученными результатами определения твердости монокристаллов $\mathrm{Al}$ двумя способами при нагружении индентора силой 0.015-1 mN [57]. При этом отмечалось, что, несмотря на хорошее согласие между значениями, даваемыми двумя методами, ОРМ систематически завышал $H$. При индентировании инденторами Берковича и Виккерса предварительно отожженного алюминия площади отпечатков, полученные с помощью сканирующего электронного микроскопа и предсказанные функцией площади, демонстрировали отклонение не более чем на $5 \%$ в диапазоне глубин от $500 \mathrm{~nm}$ до тысяч нанометров (для обеих конфигураций инденторов) [47].

Наконец, следует обратить внимание на следующее обстоятельство. Не только при нагружении $\mathrm{Al}$, но и при внедрении индентора Берковича в GaAs и Мо (рис. 8) наблюдается скачкообразная деформация, связанная предположительно с гомогенным зарожде- 
нием дислокаций в материале под индентором [4045]. Несмотря на более сложный характер поведения диаграмм нагружения при скачкообразной деформации, некоторые особенности воздействия малоамплитудных осцилляций на дефектную структуру исследуемых материалов отчетливо наблюдаются и в этом случае. Так, можно заключить, что GaAs чувствителен к осцилляциям любой амплитуды (рис. $8, b$ ), для молибдена зона чувствительности к осцилляциям лежит выше $z=1 \mathrm{~nm}$ (рис. 8,c), а для алюминия - выше $z=0.5 \mathrm{~nm}$ (рис. $8, d$ ). Впрочем есть основания полагать, что в GaAs природа скачкообразной деформации отлична от дислокационной и заключается в фазовом переходе от структуры цинковой обманки к структуре каменной соли, который происходит под действием контактного давления [58]. При этом наблюдается зависимость амплитуды первого скачка от $\mathrm{z}$ и критической силы (рис. 9).

Практическое использование метода CSM основывается на предположении о том, что амплитуда гармонических осцилляций смещения/нагрузки должна быть много меньше измеримой глубины отпечатка. Во всех коммерческих приборах (Agilent, Hysitron и т.д.) это требование выполняется, хотя и в различной степени. Например, разрешающая способность NanoIndenter ${ }^{\circledR}$ G200 составляет $0.04 \mathrm{~nm}$, что значительно меньше рекомендуемой амплитуды $z=2 \mathrm{~nm}$. Однако насколько правомерным является использование амплитуд $z \geq 2 \mathrm{~nm}$ ?

В $[7,29]$ предлагается считать, что основными источниками погрешности в CSM являются неопределенности для амплитуд смещения $\Delta h$, нагрузки $\Delta P$ и контактной жесткости $\Delta S$, вычисляемые из простых условий

$$
\begin{gathered}
P_{\mathrm{app}}=P_{\mathrm{act}}+\Delta P / 2=P_{\mathrm{act}}+\sqrt{2} \Delta P_{\mathrm{rms}}, \\
h_{\mathrm{app}}=h_{\mathrm{act}}+\Delta h / 2=h_{\mathrm{act}}+\sqrt{2} \Delta h_{\mathrm{rms}}, \\
S_{\mathrm{act}}=\frac{1}{\sqrt{2 \pi}}\left(\frac{1}{K}\right)^{1 / m} \\
\times\left[1-\left(1-\frac{2 \sqrt{2} \Delta h_{\mathrm{rms}}}{P_{\max }} S_{\mathrm{app}}\right)^{1 / m}\right] \frac{P_{\max }}{\Delta h_{\mathrm{rms}}} .
\end{gathered}
$$

Здесь индекс ,act“ обозначает фактическое значение, индекс „арр“ - кажущееся значение, индекс „,rms“ соответствует среднеквадратичному значению. На практике величины $P_{\text {app }}, h_{\mathrm{app}}, \Delta P_{\mathrm{rms}}$ и $\Delta h_{\mathrm{rms}}$ обычно измеряются наноиндентометрами напрямую. Погрешности этого типа могут быть вызваны тем, что измерительная система CSM в активном режиме регистрирует не пиковые значения $P$ и $h$, а средние величины за один цикл осцилляций; разгрузочные кривые не являются строго линейными, т.е. жесткость в этом случае определяется не как жесткость при пиковой нагрузке. И наконец, индентор может отрываться от поверхности и терять контакт с ней в течение части колебательного цикла, как в полуконтактном режиме атомно-силового микроскопа, что делает величины $P, h$ и $S$, записанные наноиндентометром, по существу бессмысленными.

Все эти обстоятельства должны учитываться в системе уравнений (8)-(10) в виде элементов коррекции, которые были рассчитаны для $\mathrm{Cu}$ [29] и использованы нами для аналогичных расчетов в $\mathrm{Al}$ (рис. 10). Из этого рисунка следует, что применение данной коррекции оправдано только для $z \leq 2 \mathrm{~nm}$, а в остальных случаях коррекция дает завышенное значение $P$ для всех $h>75 \mathrm{~nm}$. Следовательно, коррекция Оливера-Стрейдера-Фарра [29] не является панацеей и не дает корректного результата при использовании дополнительных осцилляций с $z>2 \mathrm{~nm}$.

\section{5. Заключение}

Проведенные исследования диапазона нагрузок/деформаций, при которых метод CSM можно считать „неразрушающим“ в пределах точности измерений, позволяют сделать следующие выводы.

1. Как в аморфном плавленом кварце, так и в ГЦКи ОЦК-металлах можно выделить две области специфического поведения величины $\delta h$ в зависимости от амплитуды гармонической добавки к нагружающему усилию: быстрого роста $\delta h=f(h)$ и малых вариаций около постоянной величины $\delta h \approx$ const.

2. Чем мягче и пластичнее материал, тем сильнее сказывается влияние гармонических осцилляций нагружающего усилия.

3. При любой исходной жесткости материала в диапазоне амплитуд осцилляции $z \leq 1 \mathrm{~nm}$ во всех исследованных материалах влиянием дополнительных осцилляций смещения можно пренебречь ввиду их малости.

4. С ростом амплитуды осцилляции смещения $z$ снижение твердости, определяемой в каждой точке кривой нагружения методом CSM, возрастало для GaAs, W, Mo и $\mathrm{Al}$ и было максимальным при наибольшей амплитуде осцилляции на контактных глубинах до $h_{c} \approx 300 \mathrm{~nm}$.

5. Твердость как ГЦК-, так и ОЦК-металлов уменьшалась с ростом контактной глубины, демонстрируя размерный эффект. Добавление осцилляции с амплитудой $z>1 \mathrm{~nm}$ приводит к значительному снижению твердости в наношкале, определенной как с помощью ОРМ, так и в каждой точке кривой нагружения по методу CSM. Однако с ростом $h$ это влияние постепенно снижалось и при $h$, бо́льших $\sim 500-1000 \mathrm{~nm}$, становилось меньше разброса значений $H$.

\section{Список литературы}

[1] W.C. Oliver, G.M. Pharr. J. Mater. Res. 19, 3 (2004).

[2] W.C. Oliver, G.M. Pharr. J. Mater. Res. 7, 1564 (1992).

[3] J. Hay, G. Pharr. ASM Handbook 8, 232 (2003).

[4] A.C. Fischer-Cripps. Nanoindentation. Springer, N.Y. (2011). $279 \mathrm{p}$. 
[5] Nanoindentation in materials science / Ed. J. Nemecek. In Tech, Rijeca (2012). 208 p.

[6] International Standard ISO 14577. Parts 1-4. Geneva, Switzerland (2013).

[7] J. Hay, P. Agee, E. Herbert. Experiment. Techniques 34, 3, 86 (2010).

[8] E.G. Herbert, W.C. Oliver, G.M. Pharr. J. Phys. D 41, 074021 (2008).

[9] S.R. Kohen, E. Kalfon-Kohen. Beilstein J. Nanotechnol. 4, 815 (2013).

[10] X. Li, B. Bhushan. Mater. Charact. 48, 11 (2002).

[11] W.C. Oliver, J.B. Pethica. Patent US 4848141 (1989).

[12] K.W. Siu, A.H.W. Ngan. Phil. Mag. 93, 449 (2013).

[13] S. Pathak, S.R. Kalidindi. Mater. Sci. Eng. R 91, 1 (2015).

[14] M.L.B. Palasio, B. Bhushan. Mater. Charact. 78, 1 (2013).

[15] P.J. Wei, J.F. Lin. J. Mater. Res. 24, 599 (2009).

[16] X.Y. Zhou, Z.D. Jiang, H.R. Wang, Q. Zhu. J. Phys.: Conf. Ser. 48, 1096 (2006).

[17] X. Li, B. Bhushan. Scripta Mater. 42, 929 (2000).

[18] M. Scrzypczak, C. Guerret-Piecourt, S. Bec, J.-L. Loubet, O. Guerret. J. Eur. Ceram. Soc. 29, 1021 (2009).

[19] M.D. Diop. In: Nanoindentation in material science / Ed. J. Nemecek. In Tech, Rieka (2012). P. 205-228.

[20] R. Foschia, M. Jobin, S. Hengsberger. Micron 40, 51 (2009).

[21] A. Puskar. Internal friction of materials. Cambridge International Science Publ. (2001). 328 p.

[22] Г.А. Малыгин. ФТТ 42, 69 (2000).

[23] K.W. Siu, A.H.W. Ngan. Phil. Mag. 91, 4367 (2011).

[24] K.W. Siu, A.H.W. Ngan. Mater. Sci. Eng. A 572, 56 (2013).

[25] C. Du, L. Xie. Modeling and control of vibrations in mechanical systems. CRC Press, Boca Raton (2010). 304 p.

[26] M.J. Cordill, N.R. Moody, W.W. Gerberich. J. Mater. Res. 23, 1604 (2008).

[27] M.J. Cordill, M.S. Lund, J. Parker, C. Leighten, A.K. Nair, D. Farkas, N.R. Moody, W.W. Gerberich. Int. J. Plasticity 25, 2045 (2009).

[28] M.J. Cordill, N.R. Moody, W.W. Gerberich. Inter. J. Plasticity 25, 281 (2009).

[29] G.M. Pharr, J.H. Strader, W.C. Oliver. J. Mater. Res. 24, 653 (2009).

[30] S.J. Vachhani, R.D. Doherty, S.R. Kalidindi. Acta Mater. 61, 3744 (2013).

[31] G. Guillonneau, G. Kermouche, J. Teisseire, E. Barthel, S. Bec, J.-L. Loubet. Phil. Mag. 95, 1999 (2015).

[32] G. Guillonneau, G. Kermouche, S. Bec, J.-L. Loubet. Tribology Int. 70, 190 (2014).

[33] A.H. Almasri, G.Z. Voyiadjis. J. Eng. Mater. Technol. 129, 505 (2007).

[34] E.G. Herbert, K.E. Johanns, R.S. Singleton. J. Mater. Res. 28, 3029 (2013).

[35] M. Troyon, L. Huang. J. Mater. Res. 20, 610 (2005).

[36] L. Charleux, V. Keryvin, M. Nivard, J.-P. Guin, J.-C. Sangleboeuf, Y. Yokoyama. Acta Mater. 70, 249 (2014).

[37] Ю.И. Головин. ФТТ 51, 2118 (2008).

[38] B.N. Lucas, W.C. Oliver, J.E. Swindeman. Mater. Res. Soc. Symp. Proc. 522, 3 (1998).

[39] Ю.И. Головин. Наноиндентирование и его возможности. Машиностроение, М. (2009). 312 с.

[40] D. Lorenz, A. Zeckzer, U. Hilpert, P. Grau, H. Johansen, H.S. Leipner. Phys. Rev. B 67, 172101 (2003).

[41] H.S. Leipner, D. Lorenz, A. Zeckzer, H. Lei, P. Grau. Physica B 308-310, 446 (2001).
[42] J. Li, K.J. Van Vliet, T. Zhu, S. Yip, S. Suresh. Nature 418, 307 (2002)

[43] K.J. Van Vliet, J. Li, T. Zhu, S. Yip, S. Suresh. Phys. Rev. B 67, 104105 (2003).

[44] T. Zhu, J. Li, K. Van Vliet, S. Ogata, S. Yip, S. Suresh. J. Mech. Phys. Solids 52, 691 (2004).

[45] I. Szlufarska. Mater. Today 9, 42 (2006).

[46] D.A. Lucca, K. Herrmann, M. Klopfstein. CIRP AnnalsManuf. Technol. 59, 803 (2010).

[47] T.Y. Tsui, W.C. Oliver, G.M. Pharr. Mater. Res. Soc. Symp. Proc. 436, 147 (1996).

[48] G.Z. Voyiadjis, R. Peters. Acta Mech. 211, 131 (2010).

[49] A.H. Almasri, G.Z. Voyiadjis. Acta Mech. 209, 1 (2010).

[50] Y. Liu, A.H.W. Ngan. Scripta Mater. 44, 237 (2001).

[51] G.Z. Voyiadjis, A.H. Almasri, T. Park. Mech. Res. Commun. 37, 307 (2010).

[52] N.A. Stelmashenko, M.G. Walls, L.M. Brown, Yu.V. Milman. Acta Met. Mater. 41, 2855 (1993).

[53] N.I. Tymiak, D.E. Kramer, D.F. Bahr, W.W. Gerberich. Acta Mater. 49, 1021 (2001)

[54] T. Chudoba, P. Schwaller, R. Rabe, J.-M. Breguet, J. Michler. Phil. Mag. 86, 5265 (2006).

[55] Y. Huang, F. Zhang, K.C. Hwang, W.D. Nix, G.M. Pharr, G. Feng. J. Mech. Phys. Solids 54, 1668 (2006).

[56] W.W. Gerberich, N.I. Tymiak, J.C. Grunlan, M.F. Horstemeyer, M.I. Baskes. J. Appl. Mech. 69, 433 (2002).

[57] A.V. Kulkarni, B. Bhushan. Mater. Lett. 29, 221 (1996).

[58] D. Chrobak, K. Nordlund, R. Nowak. Phys. Rev. Lett. 98, 045502 (2007). 